\title{
Multimodal Treatment of Extragonadal Choriocarcinoma with Multiple Brain and Lung Metastases: A Case Report
}

\author{
Mao Uematsu Yusuke Kanemasa Shohei Nakamura Chikako Funasaka \\ Akihiko Kageyama Tatsu Shimoyama Yasushi Omuro \\ Department of Medical Oncology, Tokyo Metropolitan Cancer and Infectious Diseases \\ Center, Komagome Hospital, Tokyo, Japan
}

\author{
Keywords \\ Germ cell tumor · Choriocarcinoma · Multimodal treatment · Brain metastases · \\ Chemoradiotherapy
}

\begin{abstract}
Choriocarcinoma is a highly aggressive germ cell tumor and can metastasize to the brain. Although brain metastasis has a poor prognosis, the optimal treatment strategy remains unclear due to its low incidence. A 33-year-old man presenting with multiple lung nodules on chest radiography was referred to our hospital. Computed tomography revealed bilateral lung nodules and a large pelvic mass, and brain magnetic resonance imaging (MRI) demonstrated multiple brain lesions. He developed progressive headache and nausea and underwent two craniotomies because of rapid tumor growth and intratumoral hemorrhage. Metastasis of choriocarcinoma was strongly suspected because of histological findings and detection of urine human chorionic gonadotropin ( $\mathrm{hCG}$ ). He immediately received chemotherapy with bleomycin, etoposide, and cisplatin (BEP). Although the pelvic mass and pulmonary lesions reduced in size and the $\beta$-hCG level decreased after one cycle of BEP, brain MRI displayed an increase in the size and number of brain metastases. He underwent whole-brain radiotherapy (WBRT) concurrently with 2 cycles of BEP, leading to successful reduction of brain metastases. After 4 cycles of BEP, the $\beta$-hCG level was still higher than the normal range, and the pelvic and pulmonary lesions remained. He continued chemotherapy with paclitaxel, ifosfamide, and cisplatin (TIP) and etoposide, ifosfamide, and cisplatin (VIP). The $\beta$-hCG level normalized, and the residual pelvic mass was resected, revealing no viable cancer cells. Multimodal treatment, including two craniotomies and chemotherapy concurrent with WBRT, can achieve good control of lesions of the brain and other sites.


Uematsu et al.: Multidisciplinary Treatment of Choriocarcinoma with Brain Metastases

\section{Introduction}

Choriocarcinoma is a highly aggressive and vascular germ cell tumor that occurs mostly in men aged 20-39 years. It has a tendency to grow rapidly, with a high risk of tumor hemorrhage and hematogenous spread to various organs. It is uniquely chemosensitive and often curable, even in cases of advanced and metastatic disease. Approximately $10 \%$ of choriocarcinomas metastasize to the brain [1]. Due to its low incidence, the optimal treatment strategies of brain metastases of choriocarcinoma remain controversial [2, 3]. Although surgery, radiation, and chemotherapy remain the mainstays of the management of brain metastases, the prognosis remains poor.

\section{Case Report}

A 33-year-old man with no significant medical history presented with incidentally detected multiple lung nodules on a chest X-ray (Fig. 1a). Computed tomography (CT) demonstrated numerous lobulated nodular densities throughout the bilateral lungs, along with a pelvic mass (Fig. 1b, c). Positron emission tomography (PET) revealed 2-deoxy-2-[ $\left.{ }^{18} \mathrm{~F}\right]-$ fluoro-d-glucose uptake in the pulmonary nodules, pelvic mass and bones, and focal defects in the brain, which suggested brain metastases (Fig. 1d, e). He was scheduled to undergo pelvic tumor biopsy but was urgently hospitalized for his progressive headache and nausea. Laboratory examination revealed highly elevated serum lactate dehydrogenase, but no other findings suggested a specific type of malignancy (Table 1). Physical examination showed a palpable mass and tenderness from the left groin to the lower abdomen and right homonymous hemianopsia. Brain magnetic resonance imaging (MRI) with contrast enhancement showed multiple metastases, especially a progressive enhancing lesion in the right cerebellum (Fig. 2a). He underwent emergency craniotomy for resection of the cerebellar lesion because of its rapid growth. Head CT on the following day displayed progressive acute parenchymal hemorrhage in the left occipital lobe tumor (Fig. 2b). He underwent a second craniotomy to control the intratumoral hemorrhage. Rapid pathological diagnosis of the cerebellar lesion showed a biphasic pattern of malignant epithelioid cells and intermixed multinucleated giant cells, which was highly suspicious of metastatic choriocarcinoma (Fig. 3).

He immediately received chemotherapy with bleomycin, etoposide, and cisplatin (BEP) after the detection of urinary human chronic gonadotropin (hCG). The size of the pelvic mass and pulmonary lesions decreased, and the $\beta$-hCG level decreased markedly to $490 \mathrm{ng} / \mathrm{mL}$ from 13,000 ng/mL after one cycle of BEP. However, brain MRI displayed an increase in the size and number of metastatic brain lesions. He underwent daily fractionated whole-brain radiotherapy (WBRT) (40 Gy in 10 fractions) concurrently with two cycles of BEP. Brain MRI after WBRT revealed a reduction in size of brain metastases. He completed 4 cycles of BEP with a favorable decrease in the $\beta$-hCG level and in the size of the pelvic mass as well as brain and pulmonary lesions. However, the $\beta$-hCG level was still higher than the normal range, and the pelvic and pulmonary lesions remained. He continued chemotherapy with paclitaxel, ifosfamide, and cisplatin (TIP) with the aim of normalizing the $\beta$-hCG level and eliminating residual tumors. Because he could not tolerate joint and muscle pain and because of leg numbness caused directly by paclitaxel, he received etoposide, ifosfamide, and cisplatin (VIP) after 4 cycles of TIP. His $\beta$-hCG level finally normalized after 3 cycles of VIP, but the residual pelvic mass was more than $1 \mathrm{~cm}$ in diameter. It was resected and was found to have no viable cancer cells (Fig. 4). He has been followed up without treatment and has shown no disease recurrence or significant neurotoxicity. 

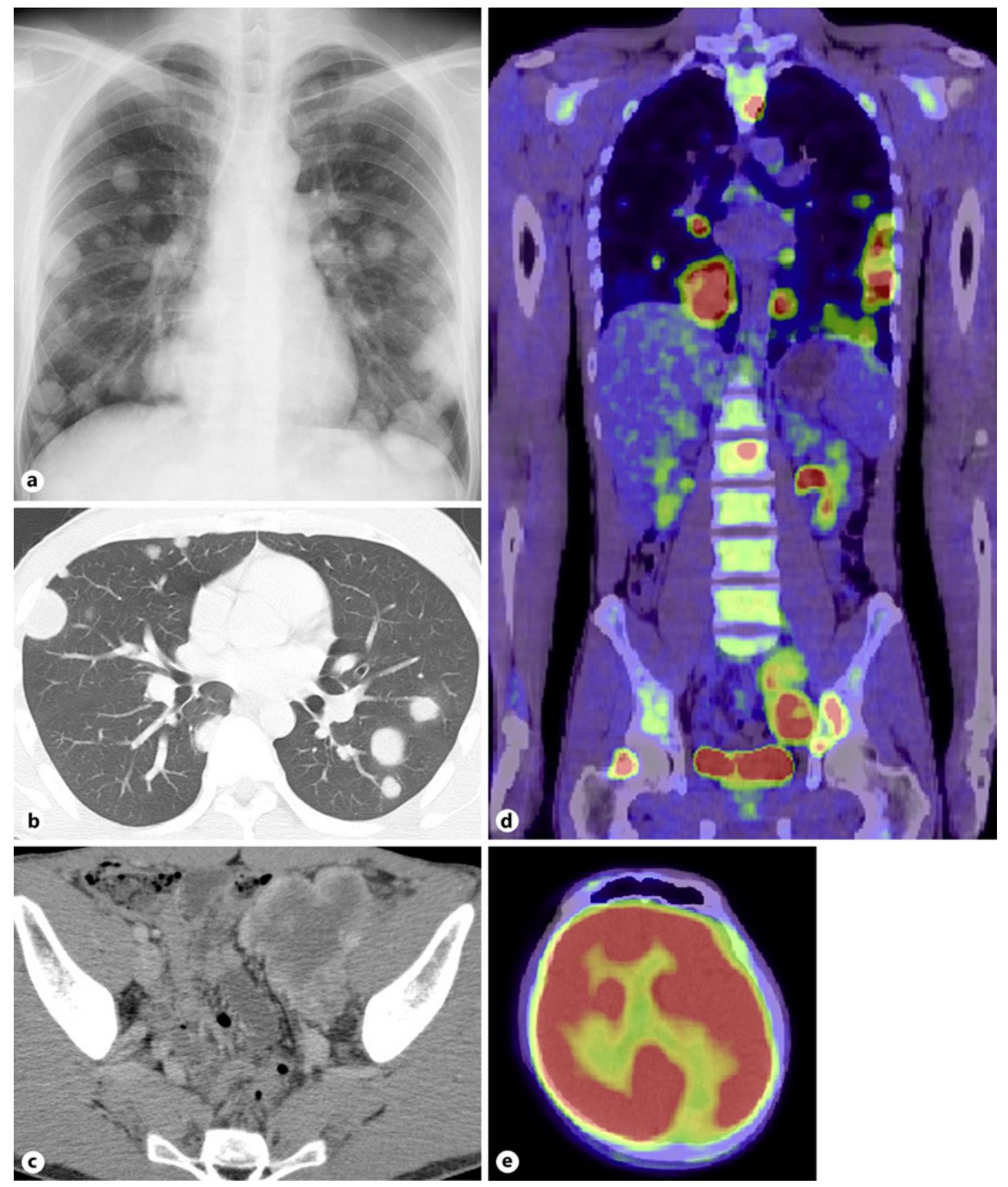

Fig. 1. Chest X-ray revealing multiple lung nodules (a). Computed tomography revealing bilateral lung nodules (b) and a pelvic mass (c). Positron emission tomography revealing 2-deoxy-2-[ $\left.{ }^{18} \mathrm{~F}\right]$-fluoro-d-glucose accumulation in lung nodules, pelvic mass, bones (d), and focal defects in the brain (e).

\section{Discussion}

Although most patients with advanced germ cell tumors can achieve long-term survival, patients with brain metastases have been considered to have poor prognoses [4]. Moreover, there is no clear consensus regarding the optimal therapeutic strategy for these patients [2, 3]. Some research groups advocate chemotherapy alone [5], multimodal treatment [6-8], or 
Table 1. Laboratory data on admission

\begin{tabular}{llllll}
\hline CBC & & & $\mathrm{Na}$ & 139 & $\mathrm{mEq} / \mathrm{L}$ \\
WBC & $12.8 \times 10^{3}$ & $/ \mu \mathrm{L}$ & $\mathrm{Cl}$ & 103 & $\mathrm{mEq} / \mathrm{L}$ \\
$\mathrm{HGB}$ & 15.0 & $\mathrm{~g} / \mathrm{dL}$ & $\mathrm{K}$ & 4.1 & $\mathrm{mEq} / \mathrm{L}$ \\
PLT & $27.4 \times 10^{4}$ & $/ \mu \mathrm{L}$ & $\mathrm{Ca}$ & 9.0 & $\mathrm{mg} / \mathrm{dL}$ \\
& & & $\mathrm{Glu}$ & 109 & $\mathrm{mg} / \mathrm{dL}$ \\
Chemistry & & $\mathrm{CRP}$ & 1.72 & $\mathrm{mg} / \mathrm{dL}$ \\
AST & 46 & $\mathrm{IU} / \mathrm{L}$ & & & \\
ALT & 94 & $\mathrm{IU} / \mathrm{L}$ & Tumor marker & & \\
LDH & 1,692 & $\mathrm{IU} / \mathrm{L}$ & $\mathrm{CEA}$ & 1.4 & $\mathrm{ng} / \mathrm{mL}$ \\
ALP & 383 & $\mathrm{IU} / \mathrm{L}$ & $\mathrm{CA} 19-9$ & 6.7 & $\mathrm{IU} / \mathrm{L}$ \\
TP & 6.4 & $\mathrm{~g} / \mathrm{dL}$ & $\mathrm{AFP}$ & 3.3 & $\mathrm{ng} / \mathrm{mL}$ \\
Alb & 3.6 & $\mathrm{~g} / \mathrm{dL}$ & $\mathrm{SCC}$ & 1.0 & $\mathrm{ng} / \mathrm{mL}$ \\
BUN & 12 & $\mathrm{mg} / \mathrm{dL}$ & ProGRP & 26.8 & $\mathrm{pg} / \mathrm{mL}$ \\
Cr & 0.69 & $\mathrm{mg} / \mathrm{dL}$ & sIL2-R & 1,000 & $\mathrm{IU} / \mathrm{L}$ \\
UA & 4.1 & $\mathrm{mg} / \mathrm{dL}$ & & & \\
\hline
\end{tabular}

CBC, complete blood count; WBC, white blood cell count; HGB, hemoglobin; PLT, platelet count; AST, aspartate aminotransferase; ALT, alanine aminotransferase; LDH, lactate dehydrogenase; ALP, alkaline phosphatase; TP, total protein; Alb, albumin; BUN, urea nitrogen; $\mathrm{Cr}$, creatinine; $\mathrm{UA}$, uric acid; $\mathrm{Na}$, sodium; $\mathrm{Cl}$, chloride; K, potassium; Ca, calcium; Glu, glucose; CRP, C-reactive protein; CEA, carcinoembryonic antigen; CA19-9, carbohydrate antigen 19-9; AFP, alpha-fetoprotein; SCC, squamous cell carcinoma; ProGRP, pro-gastrin-releasing peptide; sIL2-R, soluble interleukin 2-receptor.

even topical treatment (surgery or radiotherapy) alone [9]. According to a recent large retrospective study, the general use of surgery and/or radiation in addition to chemotherapy was not recommended for germ cell tumor patients with brain metastasis, especially not for patients with low risk factors [10]. However, we suggest that additional radiation therapy and/or neurosurgery might be beneficial in particular clinical settings according to individual characteristics.

Metastases from choriocarcinoma are frequently hemorrhagic and can lead to serious morbidity [11]. Tumor hemorrhage is a major cause of morbidity, especially in patients with brain metastases [12]. Logothetis [13] described hemorrhagic events after chemotherapy at the site of metastases containing high-volume choriocarcinomatous elements, which was termed as "choriocarcinoma syndrome." Given the high mortality associated with tumor hemorrhage and the possibility of further hemorrhage induced by chemotherapy, surgical intervention should be considered a reliable option for preventing critical cerebral hemorrhage in brain metastasis from choriocarcinoma. In this case, spontaneous tumor hemorrhage was identified before induction of chemotherapy, and thus, the patient first underwent craniotomy.

There was an increase in the size and number of metastatic brain lesions after a cycle of BEP, in spite of shrinkage of other lesions. Due to the progressive multiple brain metastases observed after surgery and chemotherapy, additional WBRT was considered for controlling the brain lesions. Because lower dose intensity of BEP has been reported to result in poor prognosis, BEP should be administered without delay whenever possible [14]. In contrast, WBRT has a risk of late neurotoxicity, including progressive leukoencephalopathy, and concurrent treatment with cranial radiotherapy and some systemic chemotherapy can increase the possibility of neurotoxicity $[5,10]$. However, in a recent study, an increase in neurotoxicity was not observed when etoposide and cisplatin were administered concurrently with cranial radiation [15]. Although the concurrent use of bleomycin and cerebral radiotherapy has not been previously reported to our knowledge, we thought that it should 

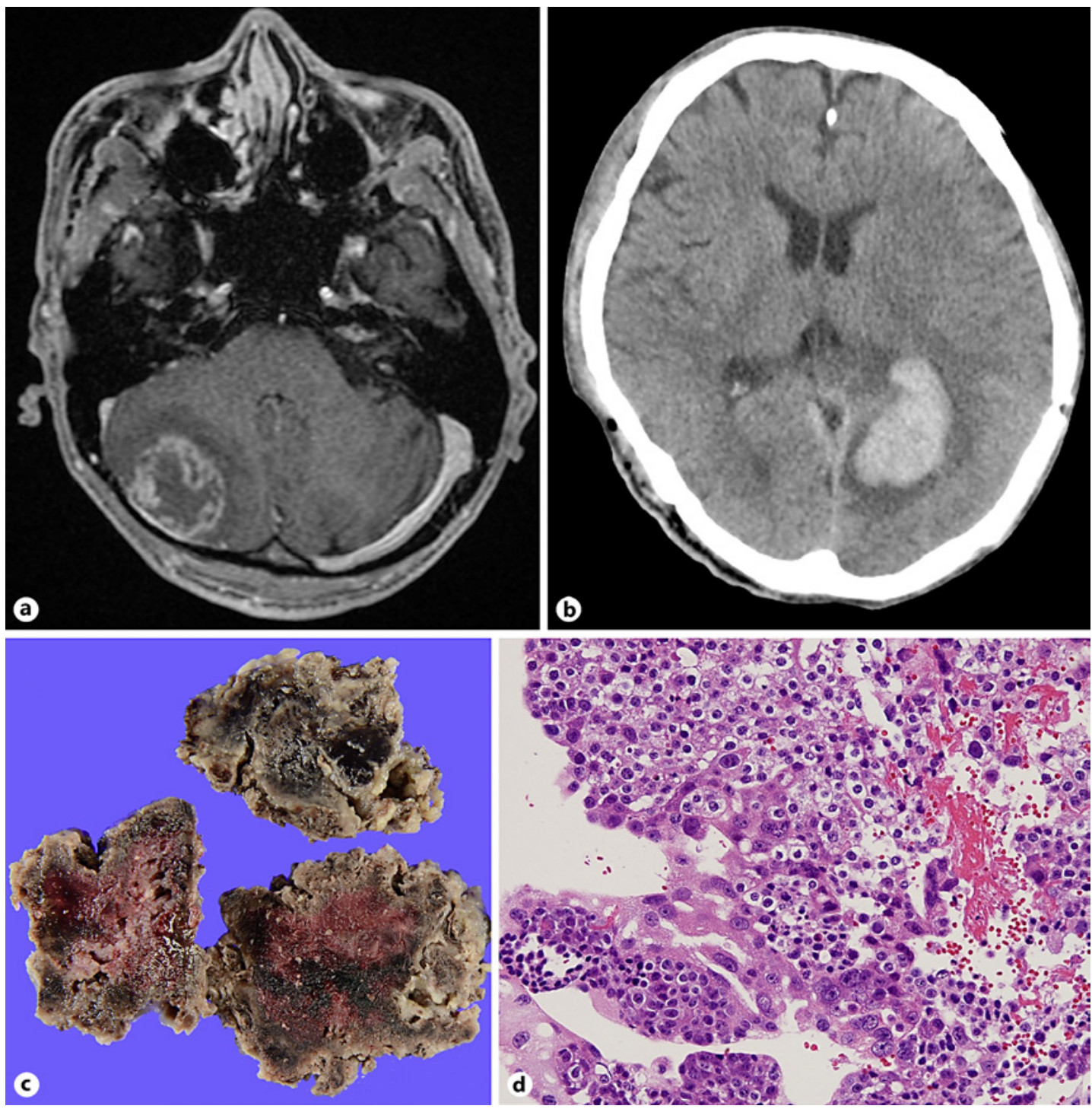

Fig. 2. Brain magnetic resonance imaging showing a contrast-enhancing lesion in the right cerebellum (a). Brain computed tomography on the day following the first craniotomy showing acute intratumoral hemorrhage in the left occipital lobe (b). Gross appearance of hemorrhagic and necrotic cerebellar masses (c). Hematoxylin-and-eosin-stained section of the tumor showing biphasic pattern of malignant epithelioid cells and intermixed multinucleated giant cells (d).

be relatively safe because of the infrequent neurotoxicity associated with bleomycin use. Actually, the patient has not experienced any late neurotoxicity for at least 1 year.

In summary, multimodal treatment, including two craniotomies and chemotherapy concurrent with WBRT, can result in good control of lesions of the brain and other sites. However, further studies are needed to confirm the efficacy and safety of this multimodal treatment.

\section{Acknowledgement}

We would like to thank Editage (www.editage.com) for English language editing. 


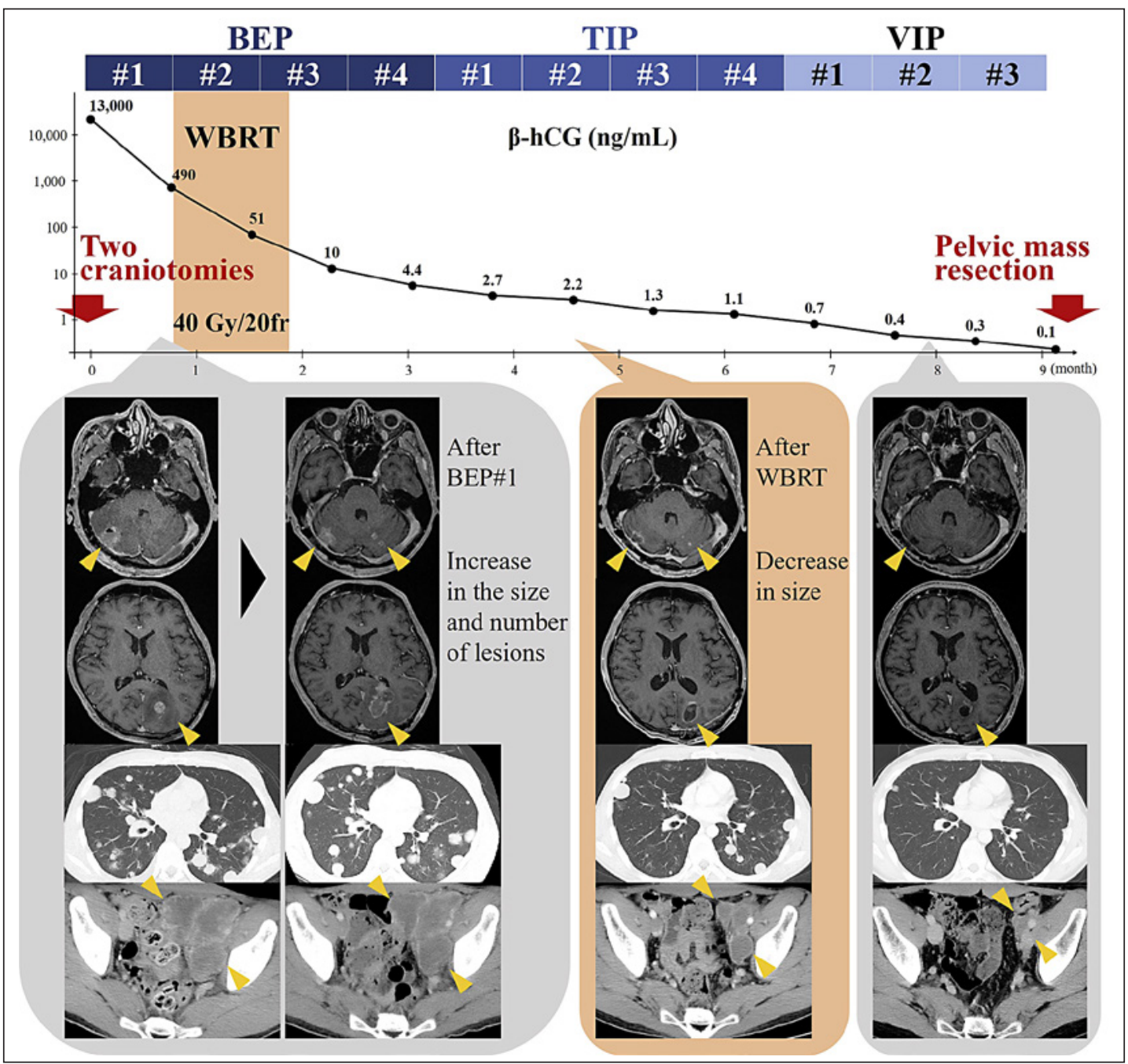

Fig. 3. Clinical course: $y$-axis showing the $\beta$-human chorionic gonadotropin ( $\beta$-hCG) level. The patient underwent whole-brain radiotherapy (WBRT) concurrently with 2 cycles of bleomycin, etoposide, and cisplatin (BEP). He continued chemotherapy with paclitaxel, ifosfamide, and cisplatin (TIP) and etoposide, ifosfamide, and cisplatin (VIP).

\section{Statement of Ethics}

We have reported this case in compliance with the Declaration of Helsinki. Informed consent was obtained from the patient for the publication of the clinical data.

\section{Disclosure Statement}

The authors have no conflicts of interest to declare.

\section{Funding Sources}

No funding was received for this research. 
Uematsu et al.: Multidisciplinary Treatment of Choriocarcinoma with Brain Metastases

\section{Author Contributions}

Mao Uematsu designed the research study, drafted the manuscript and contributed to the interpretation of data. Yusuke Kanemasa revised the manuscript and contributed to the interpretation of data. All authors treated the patient and acquired the clinical data, and have read and approved the final manuscript.

\section{References}

1 Berkowitz RS, Goldstein DP. Pathogenesis of gestational trophoblastic neoplasms. Pathobiol Annu. 1981;11: 391-411.

2 Forquer JA, Harkenrider M, Fakiris AJ, Timmerman RD, Cavaliere R, Henderson MA. Brain metastasis from non-seminomatous germ cell tumor of the testis. Expert Rev Anticancer Ther. 2007 Nov;7(11):1567-80.

3 Beyer J, Albers P, Altena R, Aparicio J, Bokemeyer C, Busch J. Maintaining success, reducing treatment burden, focusing on survivorship: highlights from the third European consensus conference on diagnosis and treatment of germ-cell cancer. Ann Oncol. 2013 Apr;24(4):878-88.

4 International Germ Cell Consensus Classification: a prognostic factor-based staging system for metastatic germ cell cancers. International germ cell cancer collaborative group. J Clin Oncol. 1997 Feb;15(2):594-603.

5 Hardt A, Krell J, Wilson PD, Harding V, Chowdhury S, Mazhar D. Brain metastases associated with germ cell tumors may be treated with chemotherapy alone. Cancer. 2014 Jun 1;120(11):1639-46.

6 Bokemeyer C, Nowak P, Haupt A, Metzner B, Kohne H, Hartmann JT. Treatment of brain metastases in patients with testicular cancer. J Clin Oncol. 1997 Apr;15(4):1449-54.

7 Fossa SD, Bokemeyer C, Gerl A, Culine S, Jones WG, Mead GM. Treatment outcome of patients with brain metastases from malignant germ cell tumors. Cancer. 1999 Feb 15;85(4):988-97.

8 Girones R, Aparicio J, Roure P, Germa-Lluch JR, Garcia Del Muro X, Vazquez-Estevez S. Synchronous versus metachronous brain metastasis from testicular germ cell tumors (TGCT): an analysis from the Spanish Germ Cell Cancer Group data base. Clin Transl Oncol. 2014 Nov;16(11):959-65.

9 Lutterbach J, Spetzger U, Bartelt S, Pagenstecher A. Malignant germ cell tumors metastatic to the brain: a model for a curable neoplasm? The Freiburg experience and a review of the literature. J Neurooncol. 2002 Jun; 58(2):147-56.

10 Feldman DR, Lorch A, Kramar A, Albany C, Einhorn LH, Giannatempo P. Brain metastases in patients with germ cell tumors: prognostic factors and treatment options-an analysis from the global germ cell cancer group. J Clin Oncol. 2016 Feb 1;34(4):345-51.

11 Motzer RJ, Bosl GJ. Hemorrhage: a complication of metastatic testicular choriocarcinoma. Urology. 1987 Aug; 30(2):119-22.

12 Mandybur TI. Intracranial hemorrhage caused by metastatic tumors. Neurology. 1977 Jul;27(7):650-5.

13 Logothetis C. Choriocarcinoma syndrome. Cancer Bull. 1984;36:118-20.

14 Toner GC, Stockler MR, Boyer MJ, Jones M, Thomson DB, Harvey VJ. Comparison of two standard chemotherapy regimens for good-prognosis germ-cell tumours: a randomised trial. Australian and New Zealand Germ Cell Trial Group. Lancet. 2001 Mar 10;357(9258):739-45.

15 Verduin M, Zindler JD, Martinussen HM, Jansen RL, Croes S, Hendriks LE. Use of systemic therapy concurrent with cranial radiotherapy for cerebral metastases of solid tumors. Oncologist. 2017 Feb;22(2):222-35. 